\title{
Umbilical hernia causing ventriculoperitoneal shunt dysfunction: illustrative cases
}

\author{
Lacey M. Carter, MD, ${ }^{1}$ Alejandro Ruiz-Elizalde, MD, ${ }^{2}$ and Naina L. Gross, $M D^{3}$ \\ ${ }^{1}$ Department of Neurosurgery and ${ }^{2}$ Division of Pediatric Surgery, Department of Surgery, University of Oklahoma Health Sciences Center, Oklahoma City, Oklahoma; and \\ ${ }^{3}$ Warren Clinic Pediatric Neurosurgery, Saint Francis Health System, Tulsa, Oklahoma
}

\begin{abstract}
BACKGROUND When ventriculoperitoneal (VP) shunts and umbilical hernias coexist in the same patient, unique complications can occur. Typically, these are readily identified problems such as cerebrospinal fluid (CSF) fistulas or entrapment of the peritoneal catheter in the hernia. The authors present cases of two children whose VP shunt dysfunction resolved after repair of their umbilical hernias.

OBSERVATIONS The authors present two cases of infant patients with shunted hydrocephalus and umbilical hernias. In both cases, the patients presented with distal shunt malfunctions not due to infection. Their shunt function improved once the umbilical hernia was repaired by pediatric surgery. Neither has required shunt revision since umbilical hernia repair.

LESSONS Although there are case reports of VP shunts eroding through the umbilicus, developing CSF fistulas, or becoming trapped inside umbilical hernias, there is no case of VP shunt dysfunction caused by just the presence of an umbilical hernia. The authors suspect that the catheter may intermittently enter and exit the hernia. This may cause intermittent obstruction of the distal catheter, or inflammation in the hernia may occur that limits CSF absorption.
\end{abstract}

https://thejns.org/doi/abs/10.3171/CASE21455

KEYWORDS ventriculoperitoneal shunt; hydrocephalus; umbilical hernia; case report

Umbilical hernias occur in 10\%-30\% of children at birth, which decreases significantly over the first 2 years of life. ${ }^{1}$ Umbilical hernias are caused by incomplete closure of the umbilical ring fascia, and only approximately $15 \%$ require surgical repair. Rarely, these common hernias have caused unique ventriculoperitoneal (VP) shunt complications, including perforations and cerebrospinal fluid (CSF) fistulas. The dynamics of peritoneal hernias, particularly umbilical hernias, and VP shunts have not been well studied. We present cases of two patients who had noninfectious peritoneal catheter malfunctions that resolved once the umbilical hernias were repaired, a previously unreported cause of malfunction.

\section{Illustrative Cases}

\section{Case 1}

A baby girl was born at 23 weeks' gestation and developed a grade 3 intraventricular hemorrhage that was initially treated with a subgaleal shunt and then converted to a VP shunt at 3 months of age. Two months after her shunt placement, at 5 months of age, she presented with 2 days of vomiting and reports of anisocoria at home. A shunt tap revealed a distal malfunction with no evidence of abdominal pseudocyst on abdominal ultrasound. This was her first shunt malfunction. She was noted to have a $2-\mathrm{cm}$ umbilical hernia with reducible bowel that had been present since birth. Her shunt was externalized at the abdominal entry site for suspected shunt infection. CSF cultures never showed infection, even after 7 days. At the same time as her shunt revision, pediatric surgery was performed to repair her umbilical hernia by a standard open technique. The patient did well postoperatively with no further concerns for vomiting or anisocoria. She has not needed another shunt revision so far, with her last follow-up 46 months after her revision and hernia repair. Retrospective review of her shunt series revealed the distal catheter tip was at the approximate entrance of the umbilical hernia (Fig. 1).

\section{Case 2}

A baby boy was born at full term with agenesis of the corpus callosum and had a VP shunt placed at 2 months of age due to

ABBREVIATIONS CSF = cerebrospinal fluid; VP = ventriculoperitoneal shunt. INCLUDE WHEN CITING Published October 25, 2021; DOI: 10.3171/CASE21455 SUBMITTED August 9, 2021. ACCEPTED September 16, 2021.

(c) 2021 The authors, CC BY-NC-ND 4.0 (http://creativecommons.org/licenses/by-nc-nd/4.0/). 


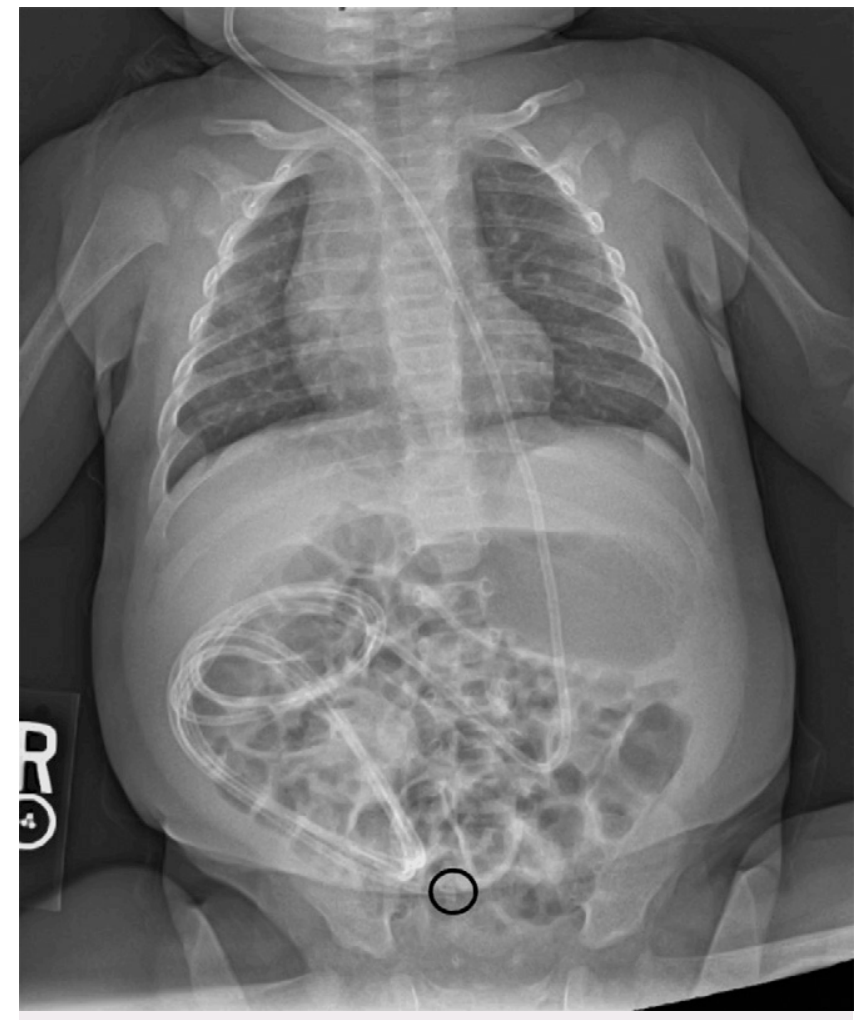

FIG. 1. Case 1: Abdominal radiograph showing tip (circle) at the approximate entrance to the umbilical hernia.

hydrocephalus manifested by increasing head circumference. One month after shunt placement, he presented with irritability and vomiting with increased head circumference. A shunt tap showed proximal flow but was equivocal for assessing distal runoff. No pseudocyst was identified on abdominal ultrasound, but the shunt was externalized at the abdominal exit site anyway for presumed shunt infection. No infection was identified on CSF cultures. After waiting 8 days to confirm negative cultures, the shunt was revised into the peritoneum again. Two weeks after surgery, the patient presented for routine postoperative follow-up. It was noted that his anterior fontanelle was full when he was supine and flat when he was upright, indicating that the shunt was likely working but not as well as would be expected. The patient's mother refused another externalization and elected careful outpatient observation. Neurosurgery requested elective repair of his umbilical hernia that had been present since birth, and this repair was performed the next week. The decision was made to cautiously observe and see how the patient did after this repair. The hernia was repaired primarily. Three weeks after hernia repair, the patient's anterior fontanelle was flat, but his head circumference increased over $3 \mathrm{~cm}$ in 2 weeks. His shunt tap showed it was working as expected. We believe this increase in head circumference occurred after his last neurosurgery visit but before his hernia repair and was not documented due to the time between clinic appointments around the hernia repair. The patient had multiple follow-up appointments with neurosurgery, which showed continued flat anterior fontanelle and improvement in his head circumference. He has not needed another shunt revision and was last seen 28 months after umbilical hernia repair. Retrospective

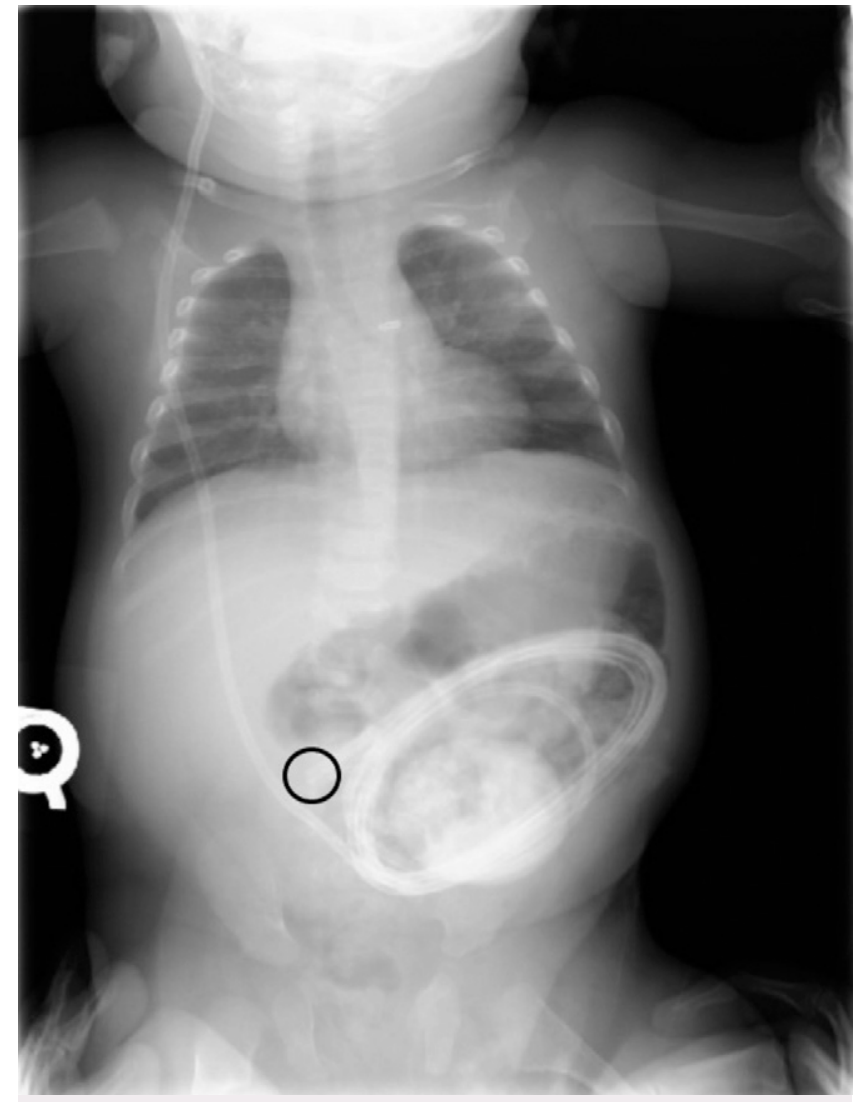

FIG. 2. Case 2: Abdominal radiograph showing tip (circle) directly inferior to the entrance to the umbilical hernia.

review of his shunt series showed the distal catheter tip was just below the entrance of the umbilical hernia (Fig. 2).

\section{Discussion \\ Observations}

There are numerous reports of VP shunt complications involving the umbilicus. These include catheter perforation through the umbilicus, entering an umbilical hernia, or creating a CSF fistula through the umbilicus. ${ }^{2-10}$ In each of these cases, the shunt was outside the body, adherent to the umbilicus, trapped in an umbilical hernia, or infected due to a fistula causing a CSF leak. Our cases are unique because the performance of our patients' VP shunts improved once their umbilical hernias were repaired without any of the reported causes of VP shunt malfunction involving the umbilicus. In both of our cases, we proved that the shunts were not infected and that the distal catheters were not entrapped in the hernia or adherent near the umbilicus.

There are more studies discussing the interaction between VP shunts and inguinal hernias. Infants with VP shunts are more likely to have inguinal complications such as hydroceles and hernias than are infants without VP shunts. ${ }^{11}$ VP shunts are known to migrate into the scrotum as the shunted fluid elevates the intraperitoneal pressure, thus distending patent processus vaginalis and inguinal hernias. ${ }^{12}$ Raised intraperitoneal pressure and increased intraperitoneal fluid are proposed reasons for the increased rate of inguinal 
hernias in shunted children. The natural history of umbilical hernias shows that any pathology that causes an increase in the intraabdominal pressure during early infancy may prolong the umbilical ring patency, leading to umbilical hernias. ${ }^{1}$ Similarly, raised intraabdominal pressure delays or even prevents closure of umbilical hernias. Although the rates of umbilical hernias in children with VP shunts have not been reported in the literature, the data on inguinal hernias combined with the natural history of umbilical hernias makes this a plausible correlation.

We do not know exactly why our observed phenomenon of distal malfunctions that improve with umbilical hernia repair occurs. One hypothesis is that the catheter intermittently enters and exits the hernia. Although we do not have imaging that shows the catheter inside of the hernia, both of our patients had abdominal radiographs that showed the shunt tip in a central location near the hernia entrance or just below it. In one reported case, a 62-year-old female required two VP shunt revisions because her distal catheter had entered her umbilical hernia, causing malfunction. ${ }^{13}$ After it was surgically replaced into the peritoneum, she required a second procedure when imaging proved the catheter had reentered the umbilical hernia. That case not only proves that distal catheter migration into the umbilical hernia can cause shunt malfunction but also shows the catheter can remigrate into the hernia. In adults and older children who spend most of their time upright, catheters tend to settle lower in the abdomen or pelvis due to gravity. Infants spend a majority of the time supine or prone, which may promote catheter migration into umbilical hernias. Another hypothesis is that inflammation occurs in the hernia or surrounding peritoneum, which decreases the amount of CSF that is absorbed. Dolas et al. suggested that sterile inflammation caused softening of the tissue around an umbilicus without obvious hernia, leading to CSF fistula. ${ }^{5}$ In two reported cases of umbilical CSF fistulas in children, umbilical hernias were believed to be contributing factors in the formation of the fistula, and the authors emphasized that the hernia must be repaired to successfully treat the fistula. ${ }^{2}$ Another study reported that umbilical hernias increase the risk of CSF fistula formation or shunt catheter extrusion through the umbilicus and even recommended trying to repair the umbilical hernia before shunt placement if possible. ${ }^{3}$ Other cases have shown that only a mild amount of inflammation in the peritoneum can cause enough of a decrease in CSF absorption to cause VP shunt malfunctions. ${ }^{14}$ Because the umbilical hernias do not have all the usual fascial layers, they may be more prone to inflammation caused by catheter friction, CSF composition, or silicon tubing, leading to poor CSF absorption.

\section{Lessons}

In young children with VP shunts, it is possible that the presence of an umbilical hernia may cause dysfunction of the shunt for an undetermined reason. In those children with continued peritoneal catheter malfunction without infection or known cause, treatment of the umbilical hernia should be considered. The association between VP shunts and umbilical hernias merits further study.

\section{References}

1. Lassaletta L, Fonkalsrud EW, Tovar JA, Dudgeon D, Asch MJ. The management of umbilical hernias in infancy and childhood. J Pediatr Surg. 1975;10(3):405-409.

2. Antunes AC, Ribeiro TR. Spontaneous umbilical fistula from ventriculoperitoneal shunt drainage. Report of two cases. J Neurosurg. 1975;43(4):481-482.

3. Ardalan M, Rafati AH, Nejat F, El Khashab M. Umbilical hernia and ventriculoperitoneal shunt complications. Iran J Pediatr. 2011;21(1): 127-128.

4. Das PC, Radhakrishna K, Rao PL. Spontaneous umbilical fistula: a rare complication of ventriculoperitoneal shunt. J Pediatr Surg. 1993;28(4):630-631.

5. Dolas I, Apaydin HO, Yucetas SC, Ucar MD, Kilinc S, Ucler N. Cerebrospinal fluid leakage from the umbilicus: case report and literature review. Int J Surg Case Rep. 2016;20:60-62.

6. Gupta A, Ahmad FU, Kumar A, Gaikwad S, Vaishya S. Umbilical CSF fistula: a rare complication of ventriculoperitoneal shunt. Acta Neurochir (Wien). 2006;148(11):1205-1207.

7. Mohindra S, Singla N, Gupta R, Gupta SK. CSF fistula through the umbilicus following a shunt surgery: a case report and literature review. Pediatr Neurosurg. 2007;43(5):396-398.

8. Pompili A, Cianfriglia F. Umbilical fistula as a complication of ventriculo-peritoneal shunt. Surg Neurol. 1979;12(2):129-130.

9. Silav G, Tun K, Dolgun H, Unlu A, Selcuki M. The spontaneous umbilical perforation of the distal end of ventriculoperitoneal shunt. Neurochirurgie. 2002;48(2-3 Pt 1):128-130.

10. Wani AA, Ramzan A, Wani MA. Protrusion of a peritoneal catheter through the umblicus: an unusual complication of a ventriculoperitoneal shunt. Pediatr Surg Int. 2002;18(2-3):171-172.

11. Clarnette TD, Lam SK, Hutson JM. Ventriculo-peritoneal shunts in children reveal the natural history of closure of the processus vaginalis. J Pediatr Surg. 1998;33(3):413-416.

12. Bates $P$, Rajderkar D. Common and uncommon causes of ventriculoperitoneal shunt malfunction diagnosed on plain radiographs. Curr Probl Diagn Radiol. 2018;47(5):317-323.

13. Laidlaw RS, Little N. Repeated cannulation of umbilical hernia with Ventriculoperiotoneal shunt catheter. J Surg Case Rep. 2014 2014(6):rju059.

14. Diluna ML, Johnson MH, Bi WL, Chiang VL, Duncan CC. Sterile ascites from a ventriculoperitoneal shunt: a case report and review of the literature. Childs Nerv Syst. 2006;22(9):1187-1193.

\section{Disclosures}

The authors report no conflict of interest concerning the materials or methods used in this study or the findings specified in this paper.

\section{Author Contributions}

Conception and design: all authors. Acquisition of data: Carter, RuizElizalde. Analysis and interpretation of data: Gross, Carter. Drafting the article: Carter. Critically revising the article: all authors. Reviewed submitted version of manuscript: Gross, Carter. Approved the final version of the manuscript on behalf of all authors: Gross. Study supervision: Gross.

\section{Correspondence}

Naina L. Gross: Warren Clinic Pediatric Neurosurgery, Saint Francis Health System, Tulsa, OK. nlgross@saintfrancis.com. 